VP-10

\title{
Laparoscopic resection of choledochal cyst type II: A case report
}

\author{
Jae Do YANG*, Mi Rin LEE, Sung Woo AHN, Hee Chul YU
}

Department of Surgery, Jeonbuk National University Hospital, Jeonju, Korea

Introduction: Type II choledochal cyst (CC), which present as a bile duct diverticulum, is extremely rare (between $0.8 \%$ and $5 \%$ of all reported choledochal cysts).

Methods: We present our experience of laparoscopic resection of a type II CC.

Results: A 28-year-old female who admitted with abdominal ultrasound revealed CC measuring $20 \mathrm{~mm}$ below gallbladder in health medical examination. MRCP showed CC measuring $24 \times 20 \mathrm{~mm}$ in the right lateral wall of the mid third of the common bile duct. Laboratory tests were normal. In familial history, grand mother and father were died at liver and pancreatic cancer. So she worried about malignant change and wanted to operation. A scheduled laparoscopy was performed with the patient in supine position. A 12 $\mathrm{mm}$ trocar was placed in the supraumbilical area. Additional four trocars were placed. After cholecystectomy was performed, common bile duct were released and dissected, leaving a $2 \mathrm{~mm}$ stump that was clipped and completing excision of the cyst. The histological examination of the specimen showed that cystic formation with chronic inflammation The patient was discharged after 3 days without problem.

Conclusions: In conclusion, type II CC are very rare. Even if there are no symptoms and bile duct dilatation, surgical treatment should be considered because of the risk of malignancy rising with age. 